\title{
Dexamethasone induces rapid promotion of norepinephrine-mediated vascular smooth muscle cell contraction
}

\author{
TING ZHANG $^{1 *}$, WEN-LEI SHI ${ }^{1,2^{*}}$, JEFFREY G. TASKER $^{3}$, JIANG-RUI ZHOU ${ }^{1}$, \\ YUN-LI PENG $^{1}$, CHAO-YU MIAO ${ }^{4}$, YONG-JI YANG ${ }^{5}$ and CHUN-LEI JIANG ${ }^{1}$ \\ ${ }^{1}$ Department of Nautical Medicine and Laboratory of Stress Medicine, Second Military Medical University, \\ Shanghai 200433; ${ }^{2}$ Department of Neurology, Bethune International Peace Hospital, Hebei 050082, P.R. China; \\ ${ }^{3}$ Neurobiology Division, Department of Cell and Molecular Biology, Tulane University, New Orleans, LA 70118, USA; \\ Departments of ${ }^{4}$ Pharmacology and ${ }^{5}$ Biophysics, Second Military Medical University, Shanghai 200433, P.R. China
}

Received July 27, 2012; Accepted November 15, 2012

DOI: $10.3892 / \mathrm{mmr} .2012 .1196$

\begin{abstract}
The aim of the present study was to identify the rapid effect of dexamethasone (Dex) on norepinephrine (NE)-mediated contraction of vascular smooth muscle cells (VSMCs) and to establish the underlying mechanism(s). Rat VSMCs were preincubated with lipopolysaccharide to simulate acute septic shock. Myosin light chain $\left(\mathrm{MLC}_{20}\right)$ phosphorylation of VSMCs was detected by western blot analysis to observe the effects of Dex on NE-mediated contraction. Activation of the RhoA/ RhoA kinase (ROCK), extracellular signal-regulated kinase (ERK) and p38 signaling pathways was detected by western blot analysis to explore the mechanism. It was identified that Dex rapidly promoted NE-induced phosphorylation of $\mathrm{MLC}_{20}$ in VSMCs and this effect may be non-genomic. The RhoA/ROCK, ERK and p38 pathways were demonstrated to be important for the rapid effect of Dex-induced promotion of NE-mediated contraction in VSMCs. The present results indicate that Dex may rapidly reverse the hyporeactivity of vasoconstriction to $\mathrm{NE}$ in vitro and this effect may be mediated by specific non-genomic mechanisms through increased activation of the RhoA/ROCK, ERK and p38 signaling pathways.
\end{abstract}

\section{Introduction}

Vascular smooth muscle cells (VSMCs) shorten during contraction, decreasing the internal diameter of blood vessels to regulate blood flow and pressure (1). VSMC contraction and relaxation are largely mediated by phosphorylation and

Correspondence to: Dr Chun-Lei Jiang, Department of Nautical Medicine and Laboratory of Stress Medicine, Second Military Medical University, 800 Xiangyin Road, Shanghai 200433, P.R. China E-mail: cljiang@vip.163.com

*Contributed equally

Key words: dexamethasone, norepinephrine, vascular smooth muscle cell, rapid promotion, contraction dephosphorylation of the 20-kDa regulatory myosin light chain $\left(\mathrm{MLC}_{20}\right)$ at threonine-18 and serine-19 by myosin light chain kinase (MLCK) and myosin light chain phosphatase (MLCP) (2). The initial phase of contraction is mediated by a rise in intracellular calcium which results in calmodulin-dependent activation of MLCK. The sustained phase of vascular contraction is thought to involve $\mathrm{Ca}^{2+}$ sensitization mechanisms (3). The major mechanism of $\mathrm{Ca}^{2+}$ sensitization of contraction is mediated by inhibition of MLCP, leading to increased $\mathrm{MLC}_{20}$ phosphorylation and VSMC contraction. The RhoA/RhoA kinase (ROCK) pathway is hypothesized to be involved in $\mathrm{Ca}^{2+}$ sensitization. The pathway is associated with sustained vasoconstriction by phosphorylating and inhibiting MLCP, subsequently increasing $\mathrm{MLC}_{20}$ phosphorylation $(4,5)$. A previous study demonstrated that the RhoA/ROCK pathway is important for drug-induced VSMC contraction or relaxation through activation or inhibition of the pathway itself. Additional studies (6-8) have revealed that extracellular signal-regulated kinase (ERK) and p38 are involved in agonist-induced smooth muscle stimulation and activation of these signaling pathways leads to phosphorylation of caldesmon (CaD), thus increasing myosin ATPase activity and promoting VSMC contraction. Glucocorticoids (GCs) are important in the stress response and are currently utilized as anti-allergic, anti-inflammatory and immunosuppressive agents. Previously, it was widely assumed that GCs function solely through regulation of gene expression and protein synthesis, a long-term response which takes several hours or days to take biological effect. However, more recently, GCs have also been identified to exert rapid non-genomic effects on various tissues and cells. Previous studies by this research group (9-12) identified that GCs inhibit degranulation of mast cells and neutrophils and phagocytosis of macrophages via a non-genomic mechanism, to exert immunosuppressive, anti-allergic and anti-inflammatory effects. The role of GCs on the circulatory system is largely mediated by permissive regulation of VSMC contraction by catecholamine, leading to enhanced maintenance of vascular tone and blood pressure (13). In clinical practice, the pressor effect of norepinephrine (NE) alone to lower blood pressure is considered unsatisfactory, particularly during rescue therapy for cases of septic shock. Administration of a small amount of cortisol is known 
to significantly enhance the pressor effect of NE. However, the mechanism by which GCs rapidly enhance NE-mediated contraction of VSMCs remains unclear. A previous study using sepsis models, hypothesized that the mechanism of vascular hyporeactivity was associated with decreased $\mathrm{Ca}^{2+}$ sensitization of VSMCs (14). Therefore, the aim of the present study was to characterize the rapid effect of dexamethasone (Dex) in NE-mediated contraction in vitro and clarify the mechanism behind this clinically important interaction.

\section{Materials and methods}

VSMC culture. Rat vascular smooth muscle cells (A7r5 cells) were purchased from the Committee on Type Culture Collection of the Chinese Academy of Sciences (Shanghai, China). Cells were incubated with growth medium for $24 \mathrm{~h}$ and then replaced with serum-free medium. After $18 \mathrm{~h}$, cells were treated with various drugs. The study was approved by the ethics committee of the Second Military Medical University.

Protein extraction. Total protein was extracted from cells using RIPA buffer, supplemented with protease and phosphatase inhibitors. Protein concentration in the supernatant of cell lysate was measured using BCA Protein Assay kit. Following this, the protein was prepared with $5 \mathrm{X}$ sample buffer and stored at $80^{\circ} \mathrm{C}$ until use.

Western blot analysis. Protein-matched samples were electrophoresed by SDS-PAGE, transferred to PVDF membranes and blocked with 5\% non-fat milk. Membranes then were incubated with the appropriate primary antibody, followed by the corresponding secondary antibody. Membranes were developed with ECL reagents and bands were visualized and quantified using Quantity One imaging software (Bio-Rad, Hercules, CA, USA).

Solutions and materials. Solutions and materials included lipopolysaccharide (LPS), NE and Dex (Sigma, St. Louis, MO, USA); Y-27632 (generously provided by the Welfide Corp., Osaka, Japan); RIPA buffer, BCA Protein Assay kit and 5X sample buffer (Beyotime Institute of Biotechnology, Jiangsu, China); antibodies against MLC, P-MLC ${ }^{\text {Ser19 }}$, MAPK, P-MAPK (Cell Signaling Technology, Inc., Danvers, MA, USA) myosin phosphatase target subunit 1 (MYPT1) and P-MYPT1 (Santa Cruz Biotechnology, Santa Cruz, CA, USA); PVDF membrane (Millipore, Billerica, MA, USA); and ECL reagents (Pierce Biotechnology, Inc., Rockford, IL, USA).

Statistical analysis. Statistical analysis was performed using SPSS software (v17.0) using raw data. All values are expressed as mean \pm SE. Data for BP were analyzed by paired and unpaired Student's t-tests. Additional results were analyzed using one-way ANOVA. $\mathrm{P}<0.05$ was considered to indicate a statistically significant difference.

\section{Results}

Dex rapidly enhances $N E$-induced $M L C_{20}$ phosphorylation in VSMCs. Mice treated with LPS are widely accepted as an acute septic shock model. NE was oxidized and deactivated by

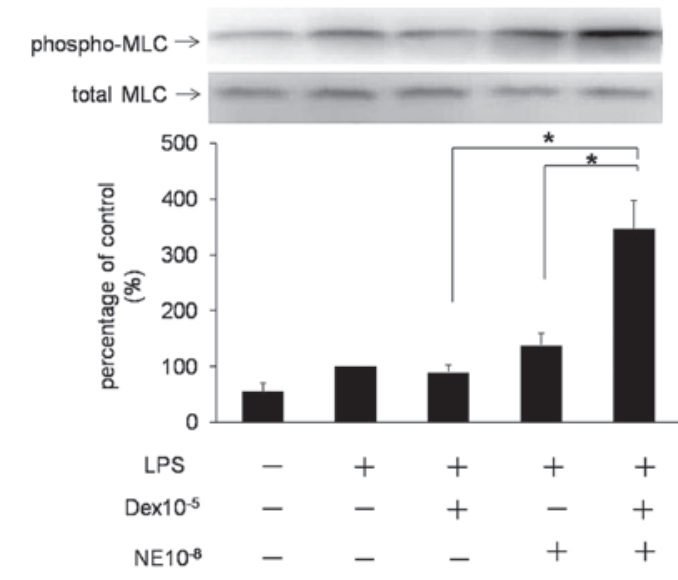

Figure 1. Regulatory effect of Dex on NE-induced phosphorylation of MLC in VSMCs. VSMCs were co-treated with LPS, Dex and NE, at $10 \mathrm{ng} / \mathrm{ml}, 10^{-5} \mathrm{M}$ for $10 \mathrm{~min}$ and $10^{-8} \mathrm{M}$ for $4 \mathrm{~min}$, respectively. Phosphorylation of MLC was detected by western blot analysis using an antibody against p-MLC. Total MLC was used to control load quantity. Bars represent p-MLC expressed as the percentage of respective controls. Results are expressed as mean $\pm \mathrm{SE}$ for 3 independent experiments; ${ }^{*} \mathrm{P}<0.05$. MLC, myosin light chain; LPS, lipopolysaccharide; Dex, dexamethasone; NE, norepinephrine; VSMCs, vascular smooth muscle cells.

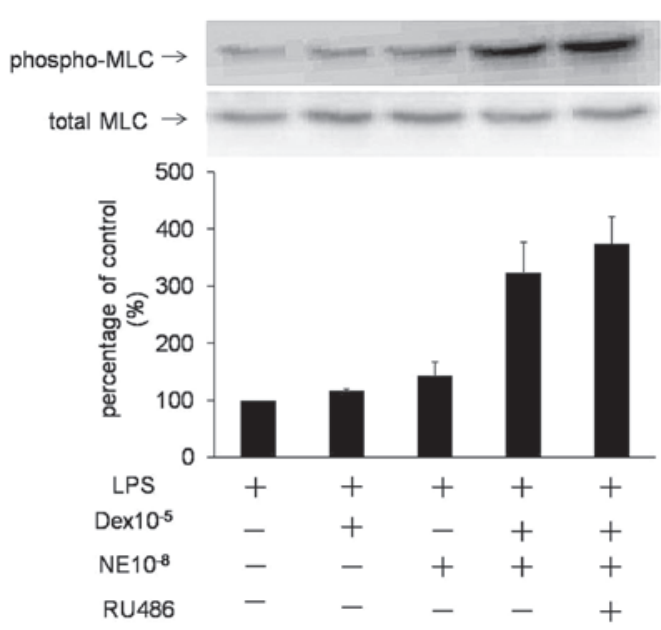

Figure 2. GR was not involved in Dex-induced promotion of NE-mediated MLC phosphorylation. VSMCs were preincubated with $10^{-6} \mathrm{M}$ RU486 for $30 \mathrm{~min}$ and then $10 \mathrm{ng} / \mathrm{ml} \mathrm{LPS}$ and $10^{-5} \mathrm{M}$ Dex were added for $10 \mathrm{~min}$. VSMCs were then treated with $10^{-8} \mathrm{M} \mathrm{NE}$ for $4 \mathrm{~min}$. Phosphorylation of MLC was detected by western blot analysis using phosphor-antibody against p-MLC. Total MLC was used to control load quantity. Bars represent the p-MLC expressed as the percentage of respective controls. Results are expressed as mean \pm SE for 3 independent experiments. GR, glucocorticoid receptor; MLC, myosin light chain; LPS, lipopolysaccharide; Dex, dexamethasone; NE, norepinephrine; VSMCs, vascular smooth muscle cells.

ONOO-1 and this deactivation induced the hyporeactivity of vasoconstriction to NE in septic shock (15). Phosphorylation of $\mathrm{MLC}_{20}$ is a key event in the activation of $\mathrm{Ca}^{2+}$-induced contraction and $\mathrm{Ca}^{2+}$ sensitization in smooth muscle (16). Fig. 1 demonstrates phosphorylation of $\mathrm{MLC}_{20}$ in VSMCs with various treatments. LPS enhanced phosphorylation of $\mathrm{MLC}_{20}$ as compared with the control group, while NE and Dex alone caused a reduced increase in the phosphorylation of $\mathrm{MLC}_{20}$ compared with the LPS group. However, preincubation with Dex enhanced phosphorylation of $\mathrm{MLC}_{20}$ in cells treated with 


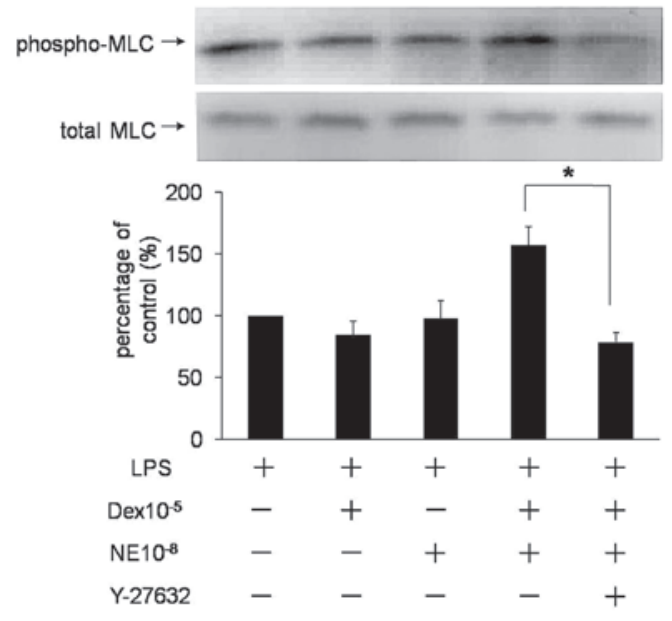

Figure 3. RhoA/ROCK activation in Dex-induced promotion of NE-mediated MLC phosphorylation. VSMCs were preincubated with $1 \mu \mathrm{M}$ Y-27632 for $30 \mathrm{~min}$ and then $10 \mathrm{ng} / \mathrm{ml} \mathrm{LPS}$ and $10^{-5} \mathrm{M}$ Dex were added for $10 \mathrm{~min}$. VSMCs were then treated with $10^{-8} \mathrm{M} \mathrm{NE}$ for $4 \mathrm{~min}$. Phosphorylation of MLC was detected by western blot analysis using an antibody against p-MLC. Total MLC was used to control load quantity. Bars represent p-MLC expressed as the percentage of respective controls. Results are expressed as mean \pm SE for 3 independent experiments; "P<0.05. MLC, myosin light chain; LPS, lipopolysaccharide; Dex, dexamethasone; NE, norepinephrine; VSMCs, vascular smooth muscle cells; ROCK, RhoA kinase.

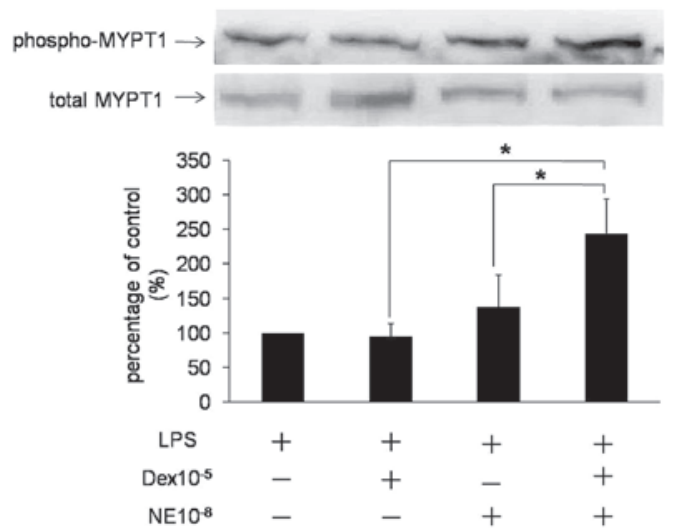

Figure 4. Activation of MYPT1 in Dex regulation of NE-mediated MLC phosphorylation. VSMCs were co-treated with $10 \mathrm{ng} / \mathrm{ml}$ LPS and $10^{-5} \mathrm{M}$ Dex for $10 \mathrm{~min}$ and then $10^{-8} \mathrm{M} \mathrm{NE}$ was added for $4 \mathrm{~min}$. Phosphorylation of MYPT1 was detected by western blot analysis using an antibody against p-MYPT1. Total MYPT1 was used to control load quantity. Bars represent p-MYPT1 expressed as the percentage of respective controls. Results are expressed as mean \pm SE for 3 independent experiments; ${ }^{*} \mathrm{P}<0.05$. MLC myosin light chain; LPS, lipopolysaccharide; Dex, dexamethasone; NE, norepinephrine; VSMCs, vascular smooth muscle cells; MYPT1, myosin phosphatase target subunit 1 .

NE $(\mathrm{P}<0.05 ; \mathrm{n}=3)$. Fig. 2 demonstrates that RU486, a GC nuclear receptor antagonist, did not block this rapid action. These results indicate that Dex rapidly enhances NE-induced $\mathrm{MLC}_{20}$ activation in VSMCs by a non-genomic mechanism.

Inhibition of Rho kinase activity reverses rapid Dex-induced promotion of NE-mediated $M L C_{20}$ phosphorylation. The RhoA/ROCK pathway participates in sustained vasoconstriction and has been proposed to be important for $\mathrm{Ca}^{2+}$ sensitization. Y-27632, a selective inhibitor of Rho kinase, was used to determine the role of Rho kinase in rapid regulation of NE-mediated

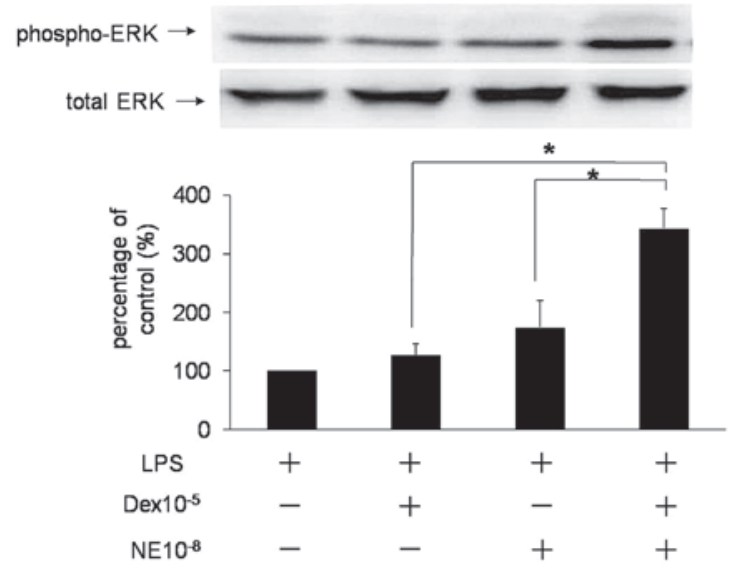

Figure 5. Activation of ERK in Dex-induced promotion of NE-mediated MLC phosphorylation. VSMCs were co-treated with $10 \mathrm{ng} / \mathrm{ml}$ LPS and $10^{-5} \mathrm{M}$ Dex for $10 \mathrm{~min}$ and then with $10^{-8} \mathrm{M} \mathrm{NE}$ for $4 \mathrm{~min}$. Phosphorylation of ERK was detected by western blot analysis using an antibody against p-ERK. Total ERK was used to control load quantity respectively. Bars represent p-ERK expressed as the percentage of respective controls. Results are expressed as mean \pm SE for 3 independent experiments; ${ }^{*} \mathrm{P}<0.05$. MLC, myosin light chain; LPS, lipopolysaccharide; Dex, dexamethasone; NE, norepinephrine; VSMCs, vascular smooth muscle cells; ERK, extracellular signal-regulated kinase.

VSMC contraction by Dex. Fig. 3 reveals that the rapid function of Dex for NE-mediated VSMC contraction $(\mathrm{P}<0.05, \mathrm{n}=3)$ was eliminated by $\mathrm{Y}-27632$, indicating that the RhoA/ROCK pathway is involved in Dex-induced rapid promotion of NE-mediated VSMC contraction.

Dex enhances NE-mediated phosphorylation of MYPT1. GTP-RhoA activates ROCK, which in turn phosphorylates MYPT1 to inactivate MLCP activity (17). Therefore, we examined whether MYPT1 ${ }^{\text {Thr853 }}$ phosphorylation accounted for NE-mediated contraction in VSMCs. Fig. 4 indicates that MYPT1 was activated by NE or Dex alone, resulting in an increase in P-MYPT1. However, when NE and Dex were administered together, phosphorylation of MYPT1 was identified to be significantly upregulated compared with NE or Dex alone $(\mathrm{P}<0.05 ; \mathrm{n}=3$; Fig. 4), indicating that Dex may enhance NE-mediated phosphorylation of MYPT1. Therefore, activation of the RhoA/ROCK pathway is involved in Dex-induced rapid promotion of NE-mediated VSMC contraction.

Dex increases NE-mediated activation of ERK. Phosphorylation of $\mathrm{CaD}$ was previously hypothesized to regulate smooth muscle contraction. Activation of ERK leads to phosphorylation of $\mathrm{CaD}$ (6). To determine whether ERK is involved in the rapid effect of Dex on NE-mediated VSMC contraction, activation of ERK was examined. Fig. 5 demonstrates that NE and Dex enhanced activation of ERK. However, when NE and Dex were administered together, the activation level of ERK was identified as significantly upregulated compared with NE or Dex alone, indicating that ERK activation occurred upstream when NE and Dex were administered together, i.e., ERK was involved in the rapid effect of Dex-induced promotion of NE-mediated VSMC contraction.

Dex increases NE-mediated activation of $p 38$. Activated p38 phosphorylates $\mathrm{CaD}$ and increases phosphorylation and 


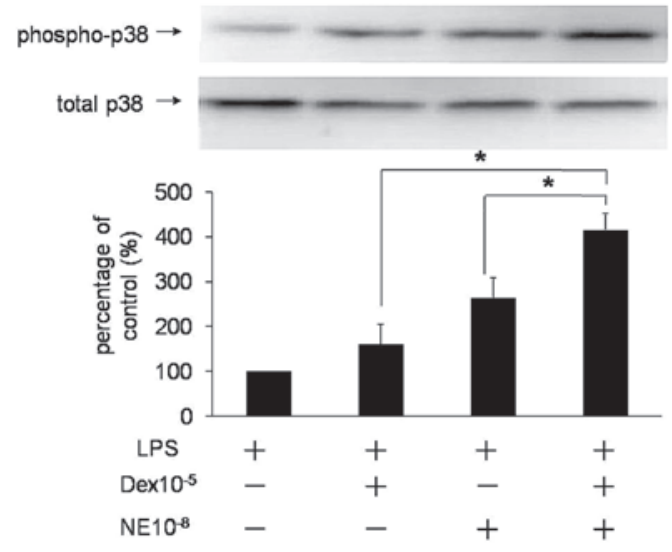

Figure 6. Activation of $\mathrm{p} 38$ in Dex-induced promotion of NE-mediated MLC phosphorylation. VSMCs were co-treated with $10 \mathrm{ng} / \mathrm{ml} \mathrm{LPS} \mathrm{and} 10^{-5} \mathrm{M}$ Dex for $10 \mathrm{~min}$ and then $10^{-8} \mathrm{M} \mathrm{NE}$ was added for $4 \mathrm{~min}$. Phosphorylation of p38 was detected by western blot analysis using an antibody against p-p38. Total p38 was used to control load quantity. Bars represent p-p38 expressed as the percentage of respective controls. Results are expressed as mean \pm SE for 3 independent experiments; "P<0.05. MLC, myosin light chain; LPS, lipopolysaccharide; Dex, dexamethasone; NE, norepinephrine; VSMCs, vascular smooth muscle cells.

activation of heat-shock protein 27 (HSP27). HSP27 is a known regulator of actin polymerization, an important event in the mechanism of force maintenance during smooth muscle contraction (8). To elucidate whether p38 was involved in NE-mediated VSMC contraction stimulated by Dex, phosphorylated p38 levels were analyzed. Results are presented in Fig. 6. A higher level of p38 activation was observed when NE and Dex were administered together. Therefore, p38, as with ERK, was identified as an additional molecule affected by Dex in NE-mediated VSMC contraction.

\section{Discussion}

Septic shock usually results in a cardiac dysfunction and a marked fall in systemic vascular resistance (18). Impaired synthesis of corticosteroids is commonly observed in the plasma of septic shock patients (19). Specific patients with sepsis and impaired adrenal function demonstrate a significant decrease in pressor sensitivity to NE, which may be improved by administration of hydrocortisone (20). In the clinic, the pressor effect of NE alone for blood pressure elevation, particularly during rescue therapy in septic shock, is considered unsatisfactory. When a small amount of cortisol is administered, the pressor effect of NE is significantly enhanced. However, the mechanism by which GCs rapidly enhance NE-mediated contraction remains unclear. In the present study, we observed the rapid effect of Dex on NE-mediated contraction in vitro and investigated the mechanism of this clinically important interaction.

Contractile activity in VSMCs is primarily determined by phosphorylation of $\mathrm{MLC}_{20}$. $\mathrm{MLC}_{20}$ phosphorylation may induce VSMC contraction directly (17). In the present study, we demonstrate a complex intracellular interaction between NE and Dex in VSMC contraction. Treatment with NE alone induced $\mathrm{MLC}_{20}$ phosphorylation and enhanced the contraction of VSMCs. Dex alone also caused low levels of $\mathrm{MLC}_{20}$ phos- phorylation. However, preincubation with Dex prior to addition of NE was identified to significantly improve the sensitivity of VSMCs to NE and enhance $\mathrm{MLC}_{20}$ phosphorylation, thus increasing the contractile effect of VSMCs. In addition, the rapid effect of Dex was not blocked by RU486, a GC nuclear receptor antagonist. The present results indicate that Dex may rapidly enhance NE-mediated contraction of VSMCs by a non-genomic mechanism.

Activation of MLCK leads to phosphorylation of $\mathrm{MLC}_{20}(17)$. In addition to MLCK, the state of $\mathrm{MLC}_{20}$ phosphorylation is regulated by MLCP, which removes the high-energy phosphate from the $\mathrm{MLC}_{20}$ to cause VSMC relaxation. As this process does not rely on $\left[\mathrm{Ca}^{2+}\right]_{\mathrm{i}}$, it is termed $\mathrm{Ca}^{2+}$ sensitization (21). The $\mathrm{Ca}^{2+}$ sensitivity of contraction is affected by variations in the ratio of MLCK/MLCP activity. A decrease in MLCP activity is likely to alter the balance in favor of MLCK, resulting in a greater degree of $\mathrm{MLC}_{20}$ phosphorylation and contraction (22).

RhoA is a well-known member of the Rho protein family (23). ROCK is the first RhoA effector. RhoA and its downstream target ROCK are important for $\mathrm{Ca}^{2+}$ sensitization (21). The activated RhoA-GTP activates ROCK, which subsequently combines with the MYPT1 subunit of MLCP to phosphorylate Thr853 and 696 sites and inhibit MLCP activity. These phosphorylation events promote the phosphorylated state of $\mathrm{MLC}_{20}$ and prolong VSMC contraction (17). The effect of ROCK is blocked by Y-27632 (24). The RhoA/ROCK-mediated pathway is critical for signal transduction initiated by a number of agonists, including NE, angiotensin II, serotonin, endothelin-1 and platelet-derived growth factor. Previous studies have indicated that the RhoA/ROCK pathway is important for numerous cellular functions, not only VSMC contraction but also actin cytoskeleton organization, cytokinesis, cell migration, proliferation and differentiation and gene expression, all of which may participate in the pathogenesis of cardiovascular disorders, including atherosclerosis, restenosis, hypertension and cardiac hypertrophy (23-27). The RhoA/ROCK pathway has also been associated with drug-induced VSMC contraction or relaxation $(28,29)$. A previous study (28) demonstrated that $\mathrm{RhoA} / \mathrm{ROCK}$ is involved in $\mathrm{Ca}^{2+}$-independent contractions induced by phorbol-12,13-dibutyrate (PDBu). PDBu phosphorylates MYPT1 by activating the RhoA/ROCK pathway, thus inactivating MLCP and causing smooth muscle contraction. An additional study (29) revealed that the RhoA/ROCK signaling pathway was involved in the regulation of vascular reactivity following hemorrhagic shock. Chiba et al (30) demonstrated that GCs inhibit airway hyperresponsiveness in allergic bronchial asthma. The mechanism of this effect involves the reduction of augmented bronchial smooth muscle contraction by GCs through inhibition of RhoA upregulation. NE stimulates $\alpha_{1}$-adrenoreceptors to produce inositol-1,4,5-triphosphate, which then releases $\mathrm{Ca}^{2+}$ that may promote the increase of $\left[\mathrm{Ca}^{2+}\right]_{\mathrm{i}}$. Following this, $\mathrm{Ca}^{2+} / \mathrm{CaM}$ activates MLCK and phosphorylates $\mathrm{MLC}_{20}$, leading to VSMC contraction. In addition, $\alpha_{1}$-adrenoreceptors activate the smooth muscle RhoA/ROCK pathway and upregulate $\mathrm{Ca}^{2+}$ sensitivity of the contractile response (31). Using the sepsis model, a previous study (14) identified that decreased $\mathrm{Ca}^{2+}$ sensitization of VSMCs was the mechanism responsible for vascular hyporeactivity. The present study demonstrated that the RhoA/ROCK pathway was involved in enhancement of Dex-induced rapid promotion 
of NE-mediated VSMC contraction. Addition of Y-27632 was observed to significantly lowered the effect of Dex-induced promotion of NE-mediated $\mathrm{MLC}_{20}$ phosphorylation, indicating that the effect of Dex on the rapid enhancement of NE-mediated contraction of VSMCs may be mediated by increased phosphorylation of $\mathrm{MLC}_{20}$ by promoting NE-mediated activation of the RhoA/ROCK pathway. In addition, the mechanism by which ROCK inhibits MLCP is through phosphorylation of the MYPT1. In the present study, activation of MYPT1 was analyzed to reveal that NE-mediated phosphorylation of MYPT1 was also increased by Dex, indicating that Dex may upregulate activation of the RhoA/ROCK pathway and promote NE-mediated VSMC contraction by enhanced activity of MYPT1.

Previous studies have identified additional mechanisms of VSMC contraction, including filament rearrangement and the ERK, p38 and protein kinase C (PKC) pathways. Firstly, actin and myosin interactions result in the initial development of force, a process similar to the function of these filaments in adhesion to attachment sites where they form a cytoskeletal scaffold that maintains tension in the absence of additional cross-bridge cycling (32). Secondly, a number of studies have identified that activated ERK and p38 are involved in agonist-induced smooth muscle stimulation. Their activation leads to $\mathrm{CaD}$ phosphorylation, which increases myosin ATPase activity and promotes VSMC contraction. In addition, p38 increases phosphorylation and activation of HSP27, a regulator of actin polymerization (6-8). Finally, PKC phosphorylates calponin $(\mathrm{CaP})$ and $\mathrm{CaD}$, subsequently increasing myosin ATPase activity and contractile response in VSMCs (33).

To determine whether ERK and p38 are involved in rapid enhancement of NE-mediated VSMC contraction by Dex, we examined phosphorylation of ERK and p38. The results demonstrated that co-treatment with Dex and NE upregulated phosphorylation of ERK and p38, consistent with the hypothesis that ERK and p38 are involved in the effect of Dex on NE-mediated VSMC contraction. The association of the PKC pathway in this mechanism was not examined in the present study.

Numerous research groups $(34,35)$ have previously reported cross talk between the RhoA/ROCK and ERK pathways or that they are associated with the same pathway. Activation of ERK is involved in angiotensin II-induced contraction of pressurized mesenteric arteries. This effect is blocked by the ROCK inhibitor Y-27632, indicating that ROCK is upstream of ERK activation (34). However, another study maintained that $\alpha_{2}$-adrenoceptor-mediated vascular contraction in the porcine palmar lateral vein involved RhoA/ROCK and ERK activation, although these were separate pathways (35). Further studies are required to clarify the correlation between RhoA/ROCK and ERK or p38 pathways.

In conclusion, the present study demonstrated that Dex rapidly reversed the hyporeactivities of vasoconstriction to $\mathrm{NE}$ in vitro and this effect may be mediated by non-genomic mechanisms by increasing activation of the RhoA/ROCK signaling pathway. In addition, we identified that ERK and p38 pathways were important for Dex-induced promotion of NE-mediated contraction in VSMCs. These results may provide insight into the mechanism of rapid enhancement of NE-mediated VSMC contraction by Dex and aid development of clinical therapies against septic shock.

\section{Acknowledgements}

The present study was supported by the National Natural Science Foundation of China (no. 30971083), International Cooperation Fund of Shanghai (no. 11410706800) and the Military Twelfth Five-Year Plan (no. BMS11J016). The authors thank co-workers at the Department of Nautical Medicine and Laboratory of Stress Medicine of the Second Military Medical University.

\section{References}

1. Somlyo AP and Somlyo AV: Signal transduction and regulation in smooth muscle. Nature 372: 231-236, 1994.

2. Kamm KE and Stull JT: The function of myosin and myosin light chain kinase phosphorylation in smooth muscle. Annu Rev Pharmacol Toxicol 25: 593-620, 1985.

3. Wickman G, Lan C and Vollrath B: Functional roles of the rho/rho kinase pathway and protein kinase $\mathrm{C}$ in the relevance regulation of cerebrovascular constriction mediated by hemoglobin: relevance to subarachnoid hemorrhage and vasospasm. Circ Res 92: 809-816, 2003.

4. Ito M, Nakano T, Erdodi F and Hartshorne DJ: Myosin phosphatase: structure, regulation and function. Mol Cell Biochem 259: 197-209, 2004.

5. Somlyo AP and Somlyo AV: Signal transduction by G-proteins, rho-kinase and protein phosphatase to smooth muscle and non-muscle myosin II. J Physiol 522: 177-185, 2000.

6. Hedges JC, Oxhorn BC, Carty M, Adam LP, Yamboliev IA and Gerthoffer WT: Phosphorylation of caldesmon by ERK MAP kinases in smooth muscle. Am J Physiol Cell Physiol 278: 718-726, 2000.

7. Yamboliev IA, Hedges JC, Mutnick JL, Adam LP and Gerthoffer WT: Evidence for modulation of smooth muscle force by the p38 MAP kinase/HSP27 pathway. Am J Physiol Heart Circ Physiol 278: 1899-1907, 2000.

8. Gorenne I, Su X and Moreland RS: Caldesmon phosphorylation is catalyzed by two kinases in permeabilized and intact vascular smooth muscle. J Cell Physiol 198: 461-469, 2004.

9. Zhou J, Li M, Liu L, Sheng CQ, Li Z, Wang Y, Zhou JR, Jiang ZP, Chen YZ and Jiang CL: A novel strategy for development of glucocorticoids through non-genomic mechanism. Cell Mol Life Sci 9: 1-10, 2010.

10. Zhou J, Liu DF, Liu C, Kang ZM, Shen XH, Chen YZ, Xu T and Jiang C: Glucocorticoids inhibit degranulation of mast cells in allergic asthma via nongenomic mechanism. Allergy 63: 1177-1185, 2008.

11. Sun HW, Miao CY, Liu L, Zhou J, Su DF, Wang YX and Jiang CL: Rapid inhibitory effect of glucocorticoids on airway smooth muscle contractions in guinea pigs. Steriods 71: 154-159, 2006.

12. Liu L, Wang YX, Zhou J, Long F, Sun HW, Liu Y, Chen YZ and Jiang CL: Rapid nongenomic inhibitory effects of glucocorticoids on human neutrophil degranulation. Inflam Res 54: 37-41, 2005.

13. Yang S and Zhang L: Glucocorticoids and vascular reactivity. Curr Vasc Pharmacol 2: 1-12, 2004

14. Mansart A, Bollaert PE, Giummelly P, Capdeville-Atkinson C and Atkinson J: Effects of dexamethasone and L-canavanine on the intracellular calcium-contraction relation of the rat tail artery during septic shock. Am J Physiol Heart Circ Physiol 291: H1177-H1182, 2006.

15. Takakura K, Xiaohong W, Takeuchi K, Yasuda Y and Fukuda S: Deactivation of norepinephrine by peroxynitrite as a new pathogenesis in the hypotension of septic shock. Anesthesiology 98: 928-934, 2003.

16. Jeon SB, Jin F, Kim JI, Kim SH, Suk K, Chae SC, Jun JE, Park WH and Kim IK: A role for rho kinase in vascular contraction evoked by sodium fluoride. Biochem Biophys Res Commun 343: 27-33, 2006.

17. Somlyo AP and Somlyo AV: $\mathrm{Ca}^{2+}$ sensitivity of smooth muscle and nonmuscle myosin: modulated by $G$ proteins, kinases andmyosin phosphatase. Physiol Rev 83: 1325-1358, 2003.

18. Mansart A, Bollaert PE, Seguin C, Levy B, Longrois D and Mallie JP: Hemodynamic effects of early versus late glucocorticoid administration in experimental septic shock. Shock 19: $38-44,2003$ 
19. Zaloga GP and Marik P: Hypothalamic-pituitary-adrenal insufficiency. Crit Care Clin 17: 25-41, 2001.

20. Annane D, Bellissant E, Sebille V, Lesieur O, Mathieu B, Raphael JC and Gajdos P: Impaired pressor sensitivity to noradrenaline in septic shock patients with and without impaired adrenal function reserve. Br J Clin Pharmacol 46: 589-597, 1998

21. Hilgers RH and Webb RC: Molecular aspects of arterial smooth muscle contraction: focus on Rho. Exp Biol Med 230: 829-835, 2005.

22. Ihara E, Moffat L, Ostrander J, Walsh MP and Macdonald JA: Characterization of protein kinase pathways responsible for $\mathrm{Ca}^{2+}$ sensitization in rat ileal longitudinal smooth muscle. Am J Physiol Gastrointest Liver Physiol 293: 699-710, 2007.

23. Loirand G, Guerin P and Pacaud P: Rho kinases in cardiovascular physiology and pathophysiology. Circ Res 98: 322-334, 2006

24. Noma K, Oyama N and Liao JK: Physiological role of ROCKs in the cardiovascular system. Am J Physiol Cell Physiol 290: C661-C668, 2006.

25. Shimokawa $\mathrm{H}$ and Takeshita A: Rho-kinase is an important therapeutic target in cardiovascular medicine. Arterioscler Thromb Vasc Biol 25: 1767-1775, 2005.

26. Hirokazu T, Hiroki C, Tadashi I, Jun K, Haruhiko M, Yoshisato T, Miyako T, Kazuhiko Y, Masaki N and Hisashi S: Diverse activation states of RhoA in human lung cancer cells: contribution of $\mathrm{G}$ protein coupled receptors. Int J Oncol 30: 709-715, 2007.

27. Bellizzi A, Mangia A, Chiriatti A, Petroni S, Quaranta M, Schittulli F, Malfettone A, Cardone RA, Paradiso A and Reshkin SJ: RhoA protein expression in primary breast cancers and matched lymphocytes is associated with progression of the disease. Int J Mol Med 22: 25-31, 2008
28. Baek I, Jeon SB, Kim J, Seok YM, Song MJ, Chae SC, Jun JE, Park WH and Kim IK: A role for Rho-kinase in $\mathrm{Ca}^{2+}$ independent contractions induced by phorbol-12,13-dibutyrate. Clin Exp Pharmacol Physiol 36: 256-261, 2009.

29. Li T, Liu L, Liu J, Ming J, Xu J, Yang G and Zhang Y: Mechnisms of Rho kinase regulation of vascular reactivity following hemorrhagic shock in rats. Shock 29: 65-70, 2008.

30. Chiba Y, Goto K, Hirahara M, Sakai $H$ and Misawa $M$ : Glucocorticoids ameliorate antigen-induced bronchial smooth muscle hyperresponsiveness by inhibiting upregulation of RhoA in rats. J Pharmacol Sci 106: 615-625, 2008.

31. Berridge MJ: Smooth muscle cell calcium activation mechanisms. J Physiol 586: 5047-5061, 2008.

32. Tseng S, Kim R, Kim T, Morgan KG and Hai CM: F-actin disruption attenuates agonist-induced $\left[\mathrm{Ca}^{2+}\right]$, myosin phosphorylation and force in smooth muscle. Am J Physiol 41: 1960-1967, 1997.

33. Tang DC, Xiang JZ and Lu WT: Calponin: a new regulatory protein for smooth muscle contraction. Prog Biochem Biophys 23: 325-329, 1996

34. Matrougui K, Tanko LB, Loufrani L, Gorny D, Levy BI, Tedgui A and Henrion D: Involvement of rho-kinase and the actin filament network in angiotensin II-induced contraction and extracellular signal-regulated kinase activity in intact rat mesenteric resistance arteries. Arterioscler Thromb Vasc Biol 21: 1288-1293, 2001.

35. Roberts RE: The role of Rho kinase and extracellular regulated kinase-mitogen-activated protein kinase in $\alpha_{2}$-adrenoceptor-mediaed vasoconstriction in the porcine palmar lateral vein. J Pharmacol Exp Ther 311: 742-747, 2004. 